\title{
EINE KONJEKTUR JOSEPH SCALIGERS ZU PHILIPPER II 30
}

\author{
VON \\ HENK JAN DE JONGE \\ Amstcrdam / Leiden
}

Der heute massgebliche Text von Phil. ii 30, wie unan thn in der Ausgabe von Nestle-ALANd findet, hat in wissenschaltsgeschichtlicher Hinsicht ein Schicksal gehabt, das in der über Jahrhunderte reichenden Geschichte der neutestamentlichen Textforschung wohl nur einmal vorgekommen ist. Das äusserst seltene Wort $\pi \alpha \rho \alpha \beta \circ \lambda \varepsilon y-$

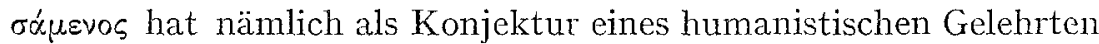
schon im I7. Jahrhundert die nach modernen Masstäben unrichtige, aber damals allgemein anerkannte Lesart des Textus receptus ersetzt und wurde erst spater durch die Handschriften bestätigt. Das Erstaunlichste an diesem Vorgang ist, dass dies Wort in dem jenem Gelchrten bekannten griechischen Wortschatz überlaupt nicht vorkam. Seine Existenz in der griechischen Sprache ist erst später bekannt geworden '). Der Mann, dem diesc Leistung gelang, ist der bedeutende Philologe und Historiker Joseph SCALIGER, der I593 bis I609 Professor ,ohne Portefeuille" an der Leidener Universität war, die 1975 ihr vichundertjähriges Bestehen feiert.

Im Wortlaut des Textus receptus von Phil. ii 30 wird die Empfehlung für Epaphroditus mit folgenden Worten begründet: "O $\tau \iota \delta\llcorner\grave{\alpha}$

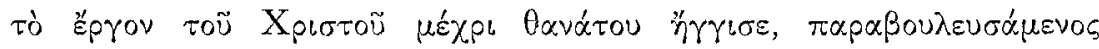
$\tau \ddot{n} \psi u \times \tilde{n}$. Das Verbum $\pi \alpha p \alpha \beta o u \lambda \varepsilon u ́ \varepsilon \sigma 0 \alpha \iota$ verstand man als Kompo-

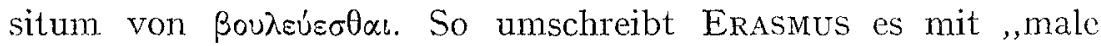
consulere, et non habere rationem alicujus". LUTIER übersetzt: „da er sein Leben geringe bedachte”, BEZA ,non habita vitae ratione”, und bemerkt dazu: „Nam $\pi \alpha \rho \alpha \beta o u \lambda \varepsilon u ́ \varepsilon \sigma \theta \alpha \iota$ significat perperam consulere, uti faciunt qui sc in apertum vitac periculum

1) S. Adolf Deissman, Lucht von Osten, Tubingen I9234, \$. 68-69: „Das

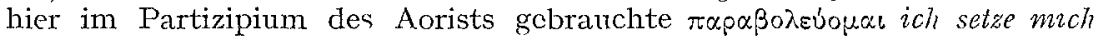
aus ist noch nicht belegt gewesen und war wohl schon in alter Zeit ein so ungewohnliches Wort, dass es von einigen Abschreibein verändert worden ist." 
coniiciunt." In diesem Sinn wurde $\pi \alpha p \alpha \beta o u \lambda \varepsilon v \sigma \alpha ́ \mu \varepsilon v o s$ auch von Arias Montanus aufgefasst (,Posthabens animam”), ebenfalls von Castellio, Flacius Illyricus, Piscator und Vatablus (oder welcher andere Verfasser die unter VATABLUs' Namen herausgegebenen Noten zum Neuen Testament geschrieben haben mag) ${ }^{2}$ ). Entsprechend lautet die Anmerkung zu Phil. ii 30 in der niederländischen „Statenbijbcl”: „Het Griecks woort betecckent raet ncmen sonder op sijn ziele, ofte leven te achten ofte letten" ${ }^{3}$ ).

Nun hatte aber Scaliger I60o in Leiden die zweite Ausgabe seiner kommentierten Manilius-Edition publiziert. In seinem Kommentar zu IV, x84 (S. 3I7) spricht er über die bezalıten Schaukämpfer, die in den römischen Theatern gegen wilde Tiere antraten. Er führt aus, dass diesc Kämpfer „propter desperatam audaciam”

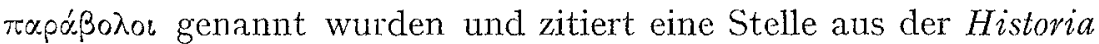
ecclesiastica von Socrates (5. Jhdt. n. Chr.), in der das Wort $\pi \alpha p \alpha ́ \beta 0 \lambda \varsigma_{s}$ als Bezeichnung für ,Wettkämpfer" belegt ist. Dem fügt er mit der ihm eigenen Kürze, Einfachheit und Selbstgewissheit zu: ,Inde verbum elegans Apostolo $\pi \alpha \rho \alpha \beta o \lambda \varepsilon v \varepsilon \sigma \theta \alpha<$; quod alij in

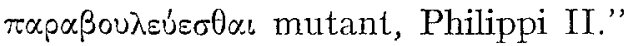

SCALIGER hat für seine Lesart $\pi \alpha \rho \alpha \beta o \lambda \varepsilon v \sigma \dot{\alpha} \mu \varepsilon v o s$ keine handschriftliche Grundlage gehabt. Weder in einem Kommentar, noch in irgendeiner Variantenlistc ${ }^{4}$ ), die vor 1600 erschienen waren, war registriert, dass in einigen Handschriften für $\pi \alpha \rho \alpha \beta o u \lambda \varepsilon v \sigma \alpha \dot{\mu} \mu \varepsilon v o s$ etwas anderes strbt. Auch das griechisch-arabische Lektionar, das Scaligrer gerade im Jahr i60o zum Gescbenk erhielt (jetzt Leiden, Universitätsbibliothek, MS Or. $243=$ Gregory Lekt. 6) ${ }^{5}$ ) enthält keine Perikope aus dem Philipperbrief, sodass es für ihn keine Hilfe bei der Rekonstruktion von Phil. ii 30 bedeutet haben kann. SCALIGER hätte aber auch sicher keine neutestamentliche Handschrift zu Rate ziehen wollen, um diese oder auch andere Korrekturen zu stützen. Zwar vertrat er die Ansicht, dass bei nichtbiblischen griechischen oder lateinischen Schriftstellern der Text mit Hilfe

2) S. Matth. PoLUs, Synopsis Criticorum aliorumque Sacrae Scripturae Lnterpretum et Commentatorum, Frankfurt I6943, V, col. $82 \mathrm{I}$ ad Phil. 2, 30.

$\left.{ }^{3}\right)$,Das griechische Wort bedeutet: sich zu etwas entschliessen, ohne auf seine Seele, cl.h. sein Leben, zu achten oder aufzupassen."

4) Z.B. Gulielmus Canter, ,Variarum Novi Testamenti Lectionum Jibellus" in der Antwerpener Polyglotte, Band VII, Antworpen 1571-72.

5) S. , Joseph Scaliger's Grcek-Arabic Lectionary", Quaerendo. A Quarterly Journal from the Low Countries devoted to Manuscripts and Printed Books, 5 (r975), r 43-r 72. 
von Handschriften (,ope codicum") und nicht konjektural (,,ex ingenio") zu konstituieren sei, und er hat diese damals keineswegs allgemein anerkannte Methode vorbildlich angewendet. Aber für das Neue Testament versprach er sich von dieser Methode keinen Erfolg, weil er die neutestamentlichen Handschriften für von Grund auf korrumpiert hielt. ,Quant aux vieux livres (=: Handschriften) du Nouveau Testament à la main, je ne m'y voudrois tenir, tant vieux soient-ils, car ils sont tres corrompus ..." ${ }^{6}$ ).

Die Genialität von Scaligers Konjektur wird jedoch erst deutlich, wenn man sich klarmacht, dass Scaliger im Jahre I60o keinen einzigen Beleg für die Existenz des Verbums $\pi \alpha \rho \alpha \beta=\lambda \varepsilon v \varepsilon \varepsilon \sigma \theta \alpha$, gekannt hat. Er hat das Wort sclbst erfunden, ebenso wie er sich - weniger glücklich - das Wort $\pi$ upia für „Brandopfer” einfach ausgedacht hat, un es in Mc. ix 49 an die Stelle von $\pi u p i$ zu setzen ${ }^{7}$ ). Der für uns Hentige aussergewöhnliche Mut, der sich in der Einführung eincr eigenen Wortschöpfung in den Text zeigt, beruht auf dem Verhältnis, das die Humanisten zu den alten Sprachen hatten: sie bildeten für sie nicht nur den Gegenstand wissenschaftlicher Forschung, sondern waren vor allem die Bildungssphäre, in der sie lebten. So fühlten sie sich berechtigt, darin auch eine Art imitativer Kreativität $7 u$ entwickeln.

Mit Recht nannle Scaliger also I60I seine Lesart $\pi \alpha \rho \alpha \beta o \lambda \varepsilon v \sigma-$

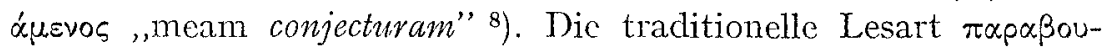
$\lambda \varepsilon v \sigma \alpha \dot{\alpha} \mu \varepsilon v \sigma \varsigma$ sah er offenbar als eine von den vielen uralten Verderbnissen des neutestamentlichen Textes an, von denen er stolz zu sagen pflegte, dass er allein sie bemerkt hätte: „Sunt ... passim

6) Scaligerana Secunda, ed. Des MAIze $\Lambda$ ux, Amsterdam I740, s.v. Testament, schon zitiert von Jac. Bernays, Joseph Justus Scaliger, Berlin I 855 (Ncudruck Osnabrück 1965), S. 203.

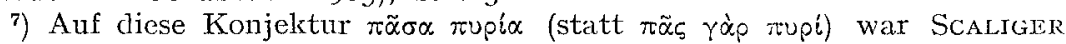
besonders stolz. Siehe seinen Brief an Servinus in den Casauboniana, Hamburg I7ro, S. 89-9o (cf. S. 289, $\Lambda \mathrm{nm}$.); seinen Brief an DE LAwT in Scaligeri Epistolae, J eiden 1627, S. 807-8; ,, Notae Iosephi Scaligeri in locos aliquot difficiliores", in Novum Iesu Christi D. N. Testamentum, Coloniae Allobrogum/Genevae $\mathrm{I} 6 \mathrm{I} 9 / 20$, S. 4 (auch in Critici sacri ad loc.); und Grotrus ad Mc ix, 49: „Mcmini virum incomparabilem Josephum Scaligerum mihi dicere mutandam lectionem, et scribendum $\pi \tilde{\alpha} \sigma \alpha[\gamma \dot{\alpha} \rho] \pi v p i \alpha$. Volebat autem rupla vocem esse novam ì. Marco confictam ad exprimendam vim vocis Hebraeae

8) Scaltger ad Casaubonum, 27 Oktober ibot, Scaligeri Épistolae cd. I627, S. I97; anch in Opuscula ed. 16ro, p. 482: „De verbo $\pi \alpha p \varepsilon \beta 0 \lambda \varepsilon u ́ \sigma \alpha \tau o$, jam memini judicij tui Et gratum est, meam conjecturam limatissimo judicio tuo confirmari." 
in textum Evangelicum ab ultima vetustate vitia admissa, quae nemo praeter me indicaverit" "9). Mit Befriedigung und einem Anflug von Spott registriert SCALIGER nach einigen Jahren (um I605) das allgemeine Erstaunen über seine Konjektur, dic doch schliesslich ein völlig unbekanntes griechisches Wort einführte: „On ne sçavoit ce que c'estoit du mot Grec, que je restituay le premier en Saint Paul" 10). Und wie er sich häufig durch ein Plagiat beleidigt fühlte, so auch jetzt: „Boulanger s'en est bien servy et d'autres sans me nommer" 11).

Bald nach der Publikation dieser Konjektur durch Scaligre berichtete ihm sein Freund Isaac Casaubonus aus Paris, dass er dic von SCALIGER vorgeschlagene Lesart ,in dem sebr alten Kodex des seligen Dupuy" gefunden habe ${ }^{12}$ ). CASAubonus meint damit den griechisch-lateinischen Codex Claromontanus (DP), der offensichtlich schon vor 594 - und nicht, wie Scrivener, KENYon, VOGELS und andere gemeint haben, erst nach BEZAS Tod (I605) -in den Besitz von Claude Dupuy (I545-r594) gekommen war.

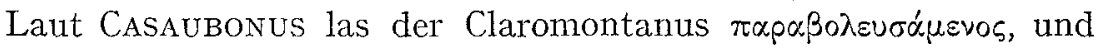
im lateinischen Text: „Parabolanus fuit de anima sua.” Hier ist das lateinische Zitat des Casaubonus ungenau. Nahczu 40 Jahre

9) Scaliger ad De LaEt, 23 Januar 1606, Scaligeri Eprstolae ed. i627, S. 808. Ibid., S. 807: „Vet ustissima menda illum [locum] occupavit: quam nemo odoratus est, quun tamen lectio vulgaris ineptissima sit."

10) Scaligerana Secunda, ed. Des Marzenux, S. 487-8.

$\left.{ }^{11}\right)$ Ibid., S. 488. Cf. Nonvelle Biographie Universelle, 7, Paris I853, sub „,Boulenger (Jules-César), historien et littétateur français, de l'ordre cles Jésuites, né à Loudon en $\mathrm{r}_{55} 8$, mort à Cahors en $\mathrm{I}_{22} 8$. On a de lui un grand nombre d'ouvrages ...", darunter De Circo Romano ludisque circensibus, Paris $\mathrm{r}_{598}$, und De Theatro ludisque scenicis libri duo, Tricassibus 1603 (mir unzugänglich). - Cf. Scaliger ad Casaubonum, i2 Januar i6oz, Scaligeri

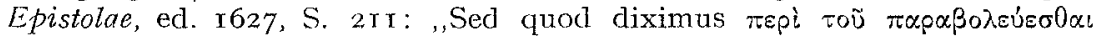
in Manilio, id omne dissimulat scarabacus ille, qui scripsit in Amphitheatrum Martialis, homo incertum imperitiorve an indignior." Der ,scarabaeus" ist offenbar Theodorus Marcilius, ,savant érudit hollandais, né à Arnheim (I548-I6I7). ... étudia les belles-lettres et le droit à Louvain, et enseigna ensuite les belles-lettres . . depuis 1578 dans divers colléges de l'nniversité de Paris; ... On a de lui une édition de Martial; Paris I 584 et r6or ...", Nouv. Biogr. Gén. 33, Paris 184 o, 503.

12) Casaubonus ad Scaligerum, um Mai i6or, Casaubone lipisl. ed. ab Almeloveen, S. I34-I 35: „Nunc narro tibi ... repcrisse me eam scripturam

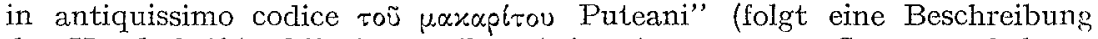
der Handschrift). Offenbar weil er keine Antwori von Scaliger bekam, wiederholte Casaubonus seine Mitteilungen in einem Brief von 8 September т601 (bei ab Almeloveen, S. 127). Cf. auch Crsauboniana, Hamburg x710, S. 83 und 135 . 
später, I639, machte SCALIGERS Nachfolger in Leiden, Claudius Salmasius, darauf aufmerksam, dass der Codex Claromontanus nicht ,parabolanus”, sondern ,,parabolatus” enthält ${ }^{13}$ ).

Vielsagend ist Scaligers Reaktion auf CaSaUbonus' Mitteilung. Er freut sich, zu hören, dass der alte Kodex von Dupuy ,parabolanus" bietet, ein Wort, das er bereits aus dem Codex Theodosianus ${ }^{14}$ ) kennt. Er stimmt Casaubonus' Meinung zu, dass an allen Stellen bei Johamnes Chrysostomus, wo $\pi \propto \rho \alpha \beta o u \lambda \varepsilon u ́ \varepsilon \sigma \theta \alpha$ vorkommt, $\pi \alpha p \alpha \beta \circ \lambda \varepsilon \dot{\varepsilon} \varepsilon \sigma \theta \alpha \iota$ gelesen werden müsse. Aber er verliert kein Wort über die Nachricht, dass der griechische Text des Codex Claromontanus seine Konjektur bestätigt. Die Lesart der Handschrift interessiert ihn überhaupt nicht. Er setzt unerschütterliches Vertrauen auf sein ingenium und auf das unfehlbare Urteil (,iudicium limatissimum") von Casaubonus, der seine Emendation billigte. Casaubonus war ja in Scaligers Augen der beste Graezist seiner Zeit. "C'est le plus grand homme que nous ayons en Grec: Je luy cede; est doctissimus omnium qui hodiè vivunt" ${ }^{15}$ ). Damit war der Fall abgeschlossen: Noch weiter etwas zugunsten seiner Konjektur zu sagen, hiesse Eulen nach Athen zu tragen. „Plura potera-

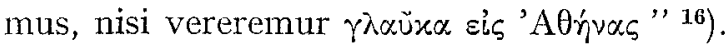

Im $x$ 7. Jahrhundert haben Lud. Capeluus, Grotius, Salmasius und andere SCALIGERs Konjektur ohne Bedenken übernommen. So wurde sie 1633 sogar in den Text der bei Elzevier in Leiden gedruckten, aber durch Whittaker zu London publizierten Ausgabe des griechischen Neuen Testaments aufgenommen. Aber SCALIGERS Vermutung wurde nicht nur durch den Claromontanus bestätigt, auch der Alexandrinus, der Sangermanensis und der Boernerianus haben diese Lesart. Und dann war sie auch im Vaticanus, Sinaiticus und Augiensis zum Vorschein gekommen, sowie in einigen Minuskelhandschriften. So hat sie schliesslich über die grossen Editionen des

19) C. Salmasius, De modo usurarum, Leiden i639, p. I98. Salmasius schreibt, dass der Claromontanus ,,parabolatus est de anima sua” hat. Auch das ist unrichtig, cl. Vetus Latina. Die Reste der altlateinuschen Bibel, nach Petrus Sabatier neu gesammelt ..., 24/2, 3. Lieferung, Freiburg i. Br. 1968, S. r77; der Claromontanus hat "parabolatus de animam suam”, Sangermanensis und Boemerianus „parabolatus de anima sua”. Kein einziger Zeuge hat die von CASAuBonus verzeichnete Lesart "parabolanus".

14) Nicht eine Bibelhandschrift, aber das Gesetzbuch. Cf. LEwIS-Short, A Latin Dictionary, s.v. , parabolanus": „Cod. Just. I, 3, r8; cf. Cod. Th. I6, 2,42 and $43 . "$

15) Scaligerana Secunda, edl. Des Maizeaux, S. 259.

16) Scaliger ad Casaubonum, 5 August i6or, Epistolae ed. I627, S. I91. 
I9. Jahrhunderts den Weg in den "Textus receptus" unserer Zeit gefunden, den Text von Nestle-Aland.

Der textkritische Apparat von Nestle-Aland führt um die 200 Konjekturen auf, von denen keine in den Text gekommen ist. Unter den Namen von mehr als 90 Gelehrten, denen die Konjekturen $z u$ verdanken sind, fehlt der von Scaliger. So ergeht es einem Gelehrten, der eine Konjektur macht, die durch die Handschriften bestätigt wird ${ }^{17}$ ).

17) Über Scalıger als Kritiker des Neuen Testaments und der ïbrigen urchristlichen Literatur siehe den Aufsatz ,The Study of the New Testament" in Leiden University in the Seventeenth Century. An Exchange of Learning, Leiden (Brill) x975, 64-Iog.

Ubrigens kann Scaliger das Verbum $\pi \alpha \rho \alpha \beta o \lambda \varepsilon v \dot{\varepsilon} \sigma 0 \alpha \iota$ auch nicht aus PsendoKallisthenes' Vita Alexandvi gekannt haben, wo es im gegenwärtigen Text (W. Kroll ed., Historia Alexandri Magni I, Berlin 1926, siehe Wortregister) einige Male vorkommt. Scaliger hat nämlich das Manuskript cler Vita Alexandri, jetzt Paris, BN gr. ${ }^{2}$ IIr, erst ${ }_{1} 602$ in Leiden leihweise einsehen können, als er es benutzte um aus Syncellus die Chronik des Eusebius zu rekonstruieren. $\Lambda$ usscrdem hat das Manuskript an den Stellen, wo Kroll $\pi \alpha \rho \alpha \beta 0 \lambda \varepsilon \dot{\varepsilon} \varepsilon \sigma \theta \alpha$ : liest, $\pi \alpha \rho \alpha \beta \circ u \lambda \varepsilon u ́ \varepsilon \sigma 0 \alpha \iota$. Die Belegstellen für $\pi \alpha \rho \alpha \beta \circ \lambda$ - und $\pi \propto p \circ \beta$ ou $\lambda \varepsilon v \varepsilon \sigma \theta \alpha \iota$ in der Vita Alexandri sind nicht verzeichnet in W. Baner's Wörterbuch zum Neuen Testament. 